\title{
Gas and Dust in the Extremely Red Object ERO J164502+4626.4
}

\author{
Thomas R. Greve \\ Institute for Astronomy, University of Edinburgh, Blackford Hill, Edinburgh EH9 3HJ, \\ United Kingdom \\ tgreve@roe.ac.uk \\ Rob J. Ivison \\ UK ATC, Royal Observatory, Blackford Hill, Edinburgh EH9 3HJ, United Kingdom \\ rji@roe.ac.uk \\ Padeli P. Papadopoulos \\ Department of Physics $\&$ Astronomy, University College London, Gower Street, London \\ WC1E 6BT, United Kingdom, and Sterrewacht Leiden, P. O. Box 9513, 2300 RA Leiden, \\ The Netherlands \\ pp@star.ucl.ac.uk
}

\begin{abstract}
We report the first detection of the lowest $\mathrm{CO}$ transition in a sub-millimetre bright galaxy and extremely red object (ERO) at $z=1.44$ using the Very Large Array $^{1}$. The total $J=1-0$ line luminosity of ERO J164502+4626.4 is $(7 \pm 1) \times$ $10^{10} \mathrm{~K} \mathrm{~km} \mathrm{~s}^{-1} \mathrm{pc}^{2}$, which yields a total molecular gas mass of $\sim 6 \times 10^{10} M_{\odot}$. We also present a map of the 850- $\mu$ m continuum emission obtained using SCUBA, from which we infer a far-IR luminosity and dust mass of $L_{F I R} \sim 9 \times 10^{12} L_{\odot}$ and $M_{d} \sim 9 \times 10^{8} M_{\odot}$. We find tentative evidence that the CO and sub-mm dust emission is extended over several tens of kpc. If confirmed by high-resolution imaging, this implies that EROJ164502+4626.4 is not simply a high redshift counterpart of a typical Ultra Luminous Infrared Galaxy (ULIRG).
\end{abstract}

Subject headings: galaxies: structure - galaxies: individual: (ERO J164502+4626.4) - galaxies: ISM

\footnotetext{
${ }^{1}$ The Very Large Array (VLA) is operated by the National Radio Observatory, which is a facility of the National Science Foundation, operated under cooperative agreement by Associated Universities, Inc.
} 


\section{Introduction}

Observations of CO provide one of the most powerful methods of probing the interstellar medium (ISM) in galaxies - i.e. determining the amount of molecular gas available to fuel star formation and accretion onto active galactic nuclei (AGN), the two processes believed to generate the large far-infrared (far-IR) luminosities of star-forming galaxies (Sanders et al. 1991). The first detection of CO at high redshift (Brown and vanden Bout 1991) revealed its potential to trace metal-enriched molecular gas in the early Universe. CO is thus key to our understanding of galaxy formation and evolution.

Extremely red objects (EROs - usually defined as galaxies with $R-K \gtrsim 5.3$, e.g. Moriondo, Cimatti \& Daddi 2000) constitute a bi-modal population - a combination of dusty, starbursting systems (Dey et al. 1999) and evolved ellipticals (Dunlop et al. 1996). While both types of objects appear similar in the optical/near-IR, the detection of sub-millimetre continuum emission and/or CO unambiguously pinpoints the dusty EROs. A significant fraction of the sub-mm-selected population of high-redshift dust-enshrouded starburst galaxies is believed to be associated with EROs (Smail et al. 1999). Since the advent of large-format bolometer arrays such as SCUBA (Holland et al. 1999) and MAMBO (Kreysa et al. 1998), over a hundred sources have been detected in (sub)millimetre surveys (Smail et al. 1997; Hughes et al. 1998; Bertoldi et al. 2001). In cases where optical/IR counterparts of sub-mm galaxies are available, it is found that the sources typically are distorted, multi-component systems with one or more components being an ERO (Ivison et al. 2002). The sub-mm population has a median redshift of $z \simeq 2.4$ (Chapman et al. 2003), and is widely believed to comprise the progenitors of present-day spheroids and massive ellipticals. At present, however, the molecular gas content of sub-mm selected galaxies remains largely unknown, and a systematic inventory of the molecular gas and its properties in these sources is required in order to properly address the question of their typical mass and evolutionary status. Of particular importance is the ratio of gas and dynamical masses in such objects, since it can help determine whether sub-mm galaxies are merely high-redshift analogues of local ultra luminous infra-red galaxies (ULIRGs) or massive, large-scale galaxy-formation events. The difficulties arise mainly because sub-mm selected galaxies have proved extremely difficult to identify in the optical (cf. Ivison et al. 1998, 2000). As a result only a handful of sub-mm selected galaxies have been detected in CO to date (Frayer et al. 1998, 1999; Ivison et al. 2001; Downes \& Solomon 2003; Genzel et al. 2003; Neri et al. 2003)

ERO J164502+4626.4 (Graham \& Dey 1996) was amongst the first EROs discovered by $\mathrm{Hu} \&$ Ridgway (1994). Deep near-IR and optical spectroscopy of J164502 put it at a redshift of $z=1.44$ (Graham \& Dey 1996; Dey et al. 1999), and the detection of the $\mathrm{H} \alpha$ line and the $[\mathrm{O} \mathrm{II}] \lambda 3726,3729$ doublet in its spectrum suggested that J164502 is an actively 
star-forming galaxy, possibly containing an AGN, and not an evolved elliptical at $z=2-4$ as initially suggested by $\mathrm{Hu} \&$ Ridgway (1994). The presence of large amounts of dust was inferred from sub-mm observations by Cimatti et al. (1998) and Dey et al. (1999), the latter detecting J164502 in the continuum at 450, 850 and $1350 \mu \mathrm{m}$. These observations together with detections of CO $J=2-1$ and $J=5-4$ by Andreani et al. (2000), which revealed the presence of large quantities of molecular gas, unamibiguously demonstrated that J164502 is a gas-rich dust-enshrouded starburst galaxy.

Here we present observations of the CO $J=1-0$ emission from J164502 using the VLA, providing the first detection of the lowest, and thus least excitation biased, CO line in a galaxy typical of the sub-mm population. We also present the $850-\mu \mathrm{m}$ SCUBA map of this source. Throughout this paper we have assumed $H_{0}=65 \mathrm{~km} \mathrm{~s}^{-1} \mathrm{Mpc}^{-1}, \Omega_{M}=0.3$ and $\Omega_{\Lambda}=0.7$. In this cosmology the luminosity distance of J164502 is $11.2 \mathrm{Gpc}$ and $1^{\prime \prime}$ corresponds to $9.1 \mathrm{kpc}$.

\section{Observations and data reduction}

\subsection{Very Large Array observations}

At $z=1.44$ the CO $J=1-0$ line from J164502 falls within the VLA's Q-band at $0.7 \mathrm{~cm}$. The line widths of the CO $J=5-4$ and $J=2-1$ lines were $\simeq 400 \mathrm{~km} \mathrm{~s}^{-1} \mathrm{FWHM}$ (Andreani et al. 2000), and a similar line width could be expected for the $J=1-0$ line. In order to cover the entire CO line, two adjacent $50 \mathrm{MHz}$ IF pairs (left and right circular polarisation) were centred at 47.235 and $47.285 \mathrm{GHz}$ giving $635 \mathrm{~km} \mathrm{~s}^{-1}$ of coverage. Measurements of J164502 in the radio have yielded $S_{20 \mathrm{~cm}} \leq 300 \mu \mathrm{Jy}$ and $S_{3.6 \mathrm{~cm}}=(35 \pm 11) \mu \mathrm{Jy}$ (Frayer 1996). Fitting a power law spectrum to these data points yields a spectral index of $\alpha \geq-1.25$. Adopting a more conservative spectrum with $\alpha=-0.7$, we find that the extrapolated synchroton flux at $0.7 \mathrm{~cm}$ amounts to no more than $11 \mu \mathrm{Jy}$. Furthermore, the dust contributes by less than $10 \mu \mathrm{Jy}$ at these wavelengths (Dey et al. 1999). Hence, any contribution from continuum emission is neglible and no continuum subtraction was necessary. Observations were obtained in $\mathrm{D}$ and $\mathrm{C}$ configurations (see Table 1). Due to bad weather, all the C-configuration data were of poor quality and discarded, leaving a total of $11.6 \mathrm{hr}$ of observing time on source. The primary calibrator, 3C 286, was observed at the beginning and end of each observing run in order to fix the absolute flux density scale. Every 3-4 minutes the antennas were pointed toward a phase calibrator to ensure phase coherence throughout the run. This fast-switching technique resulted in a residual phase rms, after calibration, of less than $\sigma_{\phi} \sim 20^{\circ}$. Calibration and data reduction was done using standard recipes in the NRAO $\mathcal{A} \mathcal{I P S}$ Cookbook. D-configuration data taken at different times were 
combined using DBCON, weighting each dataset by its total gridded weight.

The astrometrical uncertainty in the final CO map is given by $\sigma^{2}=\sigma_{\text {cal }}^{2}+\sigma_{\text {res }}^{2}+\sigma_{S N R}^{2}$, where $\sigma_{c a l}$ is the uncertainty of the assumed position of the phase calibrator, $\sigma_{\text {res }}$ is the residual $\mathrm{rms}$ phase error after the phase correction, and $\sigma_{S N R}$ is the positional error due to the presence of thermal noise. In our case the latter is by far the most dominant source of error and is given by $\sigma_{S N R} \simeq(F W H M / 2) / S N R$. Adopting $F W H M \simeq 3^{\prime \prime}$ and $S N R \simeq 7$ (see Fig. 1) we find the total positional error in the final CO map to be $\sim 0.2^{\prime \prime}$.

\subsection{Submillimetre observations}

Imaging data at 450 and $850-\mu \mathrm{m}$ were obtained for J164502 using the Submillimetre Common User Bolometer Array (SCUBA - Holland et al. (1999)) during periods of exceptionally low opacity at the James Clerk Maxwell Telescope (JCMT ${ }^{2}$ ) on Mauna Kea, Hawaii.

Data for J164502 were acquired during 1998 July 28-30 and 1998 September 03-05, a total integration time of $28.8 \mathrm{ks}$ using a $45^{\prime \prime}$ east-west nod/chop. In brief, data were flatfielded, corrected for atmospheric losses, edited both automatically and by hand, plotted onto an astrometric grid and flux calibrated using observations of planets and secondary calibration sources. The map was convolved with a Gaussian resulting in a resolution of $14^{\prime \prime}$.

\subsection{Near-IR Observations}

Near-IR K-band imaging was obtained of J164502 in June 2002 using the United Kingdom IR Telescope's (UKIRT ${ }^{3}$ ) UFTI imager, a $1024^{2} \mathrm{HgTeCd}$ array with $0.091^{\prime \prime}$ pixels. Nine individual frames taken during good to moderate seeing conditions were co-registered and combined yielding a total exposure time of $2700 \mathrm{~s}$. The seeing in the final image is $\sim 0.8^{\prime \prime}$.

Using 5 stars also detected in the USNO A2.0 catalogue (Monet et al. 1998), the UKIRT image was calibrated on to the USNO reference frame to within $0.2^{\prime \prime} \mathrm{rms}$. The CO map was

\footnotetext{
${ }^{2}$ The JCMT is operated by the Joint Astronomy Centre on behalf of the United Kingdom Particle Physics and Astronomy Research Council (PPARC), the Netherlands Organisation for Scientific Research, and the National Research Council of Canada.

${ }^{3}$ UKIRT is operated by the Joint Astronomy Centre on behalf of the United Kingdom Particle Physics and Astronomy Research Council (PPARC)
} 
aligned to the K-band frame using the tasks GEO and HGEOM in $\mathcal{A} \mathcal{I P S}$ resulting in a relative positional accuracy between the two of $\sim 0.3^{\prime \prime}$.

\section{Results}

In order to sensitively search for possible faint extended CO $J=1-0$ emission, at the expense of high spatial resolution, we used the compact D-configuration data tapered down using a Gaussian with a full-width of $60 \mathrm{k} \lambda$ at 30 per-cent amplitude, yielding a synthesized beam of $3.1^{\prime \prime} \times 2.8^{\prime \prime}$. The resulting CO map overlaid on the UKIRT K-band image is shown in Fig. 1. CO $J=1-0$ emission is detected at the $7 \sigma$ significance level in J164502. Assuming that we cover the entire $J=1-0$ line with the two IFs, and that we have rectangular passbands, we estimate the velocity-integrated flux-density using

$$
\int_{\Delta v} S_{\nu_{o b s}} d v=2 \Delta \nu_{I F} \frac{c}{\nu_{o b s}} \bar{S}_{C O},
$$

where $\Delta \nu_{I F}$ is the width of the IF and $\bar{S}_{C O}=\left(S_{1}+S_{2}\right) / 2$ is the average of the flux density measured in the two IFs. Using the task IMEAN to get the flux density of the source in the two IF maps we find $\bar{S}_{C O}=1.0 \pm 0.2 \mathrm{mJy}$ which yields a velocity-integrated flux density of $0.6 \pm 0.1 \mathrm{Jy} \mathrm{km} \mathrm{s}^{-1}$, where we have used $\nu_{o b s}=47.26 \mathrm{GHz}$ and an IF bandwidth of $45 \mathrm{MHz}$ instead of $50 \mathrm{MHz}$ due to bandpass rollover.

As first noted by Dey et al. (1999) using high-resolution HST WFPC2 images, J164502 in the optical has a reflected S-shaped morphology with two bright knots at each end. A comparison with Keck K-band imaging revealed that the bulk of the near-IR emission comes from the region between the two bright knots located $\sim 0.4^{\prime \prime}$ north of the brightest optical knot (Dey et al. 1999). As seen from Fig. 1, the peak intensity in the CO $J=1-0$ map coincides with the peak in the UKIRT K-band image both of which are coincident with the region of low optical emission found by Dey et al. (1999). A similar offset between the HST position and the CO $J=2-1$ emission was reported by Andreani et al. (2000). While the near-IR emission traces the dust-enshrouded stars in J164502, the optical emission corresponds to regions of low extinction, and it is therefore not surprising to see such an offset between the two types of emission. Since gas should be a good tracer of dust one would expect the $\mathrm{CO}$ to coincide with the K-band emission, as it appears to do within the limits of our astrometric errors.

A Gaussian fit to the CO data in the image plane yields a deconvolved source size of $4.5^{\prime \prime} \times 3.0^{\prime \prime}$, suggesting that even in the D-configuration we have resolved the CO emission, albeit marginally. Different weighting schemes and tapering functions were tried in order to 
see the effects on the extended emission. Using a less restrictive tapering function (FWHM= $200 \mathrm{k} \lambda$ ) and a robust parameter of 2 instead of 5 (natural weighting), thereby increasing the weight of the long baselines, the source still appeared extended although at a lower significance due to the larger noise on the longest baselines (see Figure 1). In order to confirm or dismiss the reality of the extended emission we shifted the peak of the CO emission to the phase center, and then plotted the binned real and imaginary parts of the complex visibilities as a function of baseline distance, Fig. 1. The complex visibilities are the Fourier components of the source brightness distribution. Hence, for a Gaussian source brightness distribution the real part of the complex visibilities is a Gaussian while the imaginary part will be zero since a Gaussian is an even function. The dashed curves in Fig. 1 represents a least-squares fit of a circular Gaussian to the visibilities. The position of the Gaussian was fixed to the phase center during the fit and only the amplitude and width were allowed to vary. We find that the real part of the visibilities is consistent with a Gaussian source model with a $F W H M=3.9^{\prime \prime}$ in agreement with the Gaussian fit in the image plane. The imaginary part is consistent with zero at all baselines within the limits of our residual rms phase error of $\sigma_{\phi} \sim 20^{\circ}$. The phase error would give rise to a dispersion around zero of $\sigma(\operatorname{Im}(V))=\sigma(S \sin \phi) \simeq S \sigma(\phi)=1.0 \mathrm{mJy}\left(20^{\circ} / 180^{\circ}\right) \pi \simeq 0.3 \mathrm{mJy}$ which is close to the observed scatter.

The $850-\mu$ m SCUBA map of J164502 is shown in Fig. 2, and is seen to contain at least two statistically significant sources. A $7 \sigma$ emission feature showing in the map is detected at a position which coincides with the near-IR and CO-detections of J164502, and we take this to unambiguously be the $850-\mu \mathrm{m}$ SCUBA detection of J164502. Another source, marked as J164502-SMM1 in Fig. 2, detected at the $5 \sigma$ level is seen $50^{\prime \prime}$ to the south, with faint emission at the $4 \sigma$ level also seen just $\sim 20^{\prime \prime}$ south of J164502. We estimated the 850- $\mu$ m flux density in four different ways. Two estimates were made by measuring the flux within apertures of $20^{\prime \prime}$ and $16^{\prime \prime}$ diameter. A third measurement was made by fitting a Gaussian to the emission, and a fourth by fitting a Gaussian with a fixed FWHM of $15^{\prime \prime}$ to the emission. The values derived from each of these measurements are given in Table 2 for J164502 as well as J164502SMM1. For J164502 we find $S_{850 \mu \mathrm{m}}=8 \pm 2 \mathrm{mJy}$ where we have adopted the average of the four different measurements. While this is in good agreement with the $850-\mu$ m measurement by Cimatti et al. (1998), it is larger than the flux quoted by Dey et al. (1999). Both of these measurements were obtained using SCUBA in its photometry mode but the latter value is based on twice as large a data-set as the former, taken in excellent weather conditions and is therefore the most reliable estimate. If this is the case, the large discrepancy between the flux density derived from our map and the value derived from photometry-measurement by Dey et al. (1999) could be due to extended emission from J164502 which would have been missed by the single pixel measurement. 
From the 850- $\mu \mathrm{m}$ map in Fig. 2 it is seen that the emission from J164502 does in fact appear to be extended, with emission to the NE. The reality of this extended emission is strengthened by the high-resolution Keck K-band image obtained by Dey et al. (1999) which shows an emission feature extending in the same direction, albeit on a smaller scale of $\gtrsim 1^{\prime \prime}$ (see Fig. 2 of Dey et al. (1999)). However, the fact that the stars traced by the K-band emission is extended on scales of $\gtrsim 9 \mathrm{kpc}$ means that cold dust is distributed on at least the same scales, and likely on much larger scales. Fitting a Gaussian to the SCUBA image yields a source size of $15.1^{\prime \prime}$ along the major axis, which is comparable to the $14^{\prime \prime}$ resolution of the map, though very tentatively suggestive of a slight extension. In Fig. 2 we compare the azimuthally averaged radial profiles of J164502 and J164502-SMM1 with the point spread function (PSF) of SCUBA at 850- $\mu$ m derived from a beam map of a bright blazar obtained during the same observing run. The radial profile of J164502 agrees with the PSF out to $r \simeq 20^{\prime \prime}$ beyond which it displays excess emission over that of a point source. This bump in the radial profile is due to the $4 \sigma$ emission feature $20^{\prime \prime}$ south $(\sim 180 \mathrm{kpc})$ of J164502. The reality of this emission feature is questionable but if real its close vicinity to J164502 could mean that it is a system in the process of merging with J164502. While there is thus tentative evidence that J164502 is extended, the large beam size of SCUBA does not allow for a firm conclusion on this issue.

For comparison we note that J164502-SMM1 is consistent with the PSF at all radii. The SCUBA $850-\mu \mathrm{m}$ beam is too large to make a reliable identification of J164502-SMM1 with an near-IR/optical source. If J164502-SMM1 is a dusty system at the same redshift as J164502 one might expect that it too would shine in CO. No CO emission was detected at the position of J164502-SMM1, however, and we set an upper limit on the velocity-integrated line flux using the equation $S_{\mathrm{CO}} \Delta v<3 \sigma(\delta v d v)^{1 / 2}$, where $\sigma$ is the rms noise, $\delta v$ the velocity resolution, and $d v$ the line width which we assume to be equal to the $J=2-1$ line width, i.e. $\sim 400 \mathrm{~km} \mathrm{~s}^{-1}$ (Andreani et al. 2000). The measured rms noise in the image is $\sigma \simeq$ $0.1 \mathrm{mJy}$ beam ${ }^{-1}$ which yields $S_{\mathrm{CO}} \Delta v<0.2 \mathrm{Jy} \mathrm{km} \mathrm{s}^{-1}$ for a velocity- coverage of $635 \mathrm{~km} \mathrm{~s}^{-1}$. The failure to detect CO-emission from J164502-SMM1 does not rule out a association since cluster velocity dispersions can go up to $\gtrsim 1000 \mathrm{~km} \mathrm{~s}^{-1}$, and in some cases even closely associated CO-emitting regions can have velocity differences of $\sim 1000 \mathrm{~km} \mathrm{~s}^{-1}$ as was seen in the high redshift radio galaxy 4C 60.07 (Papadopoulos et al. 2000). The probability of finding by chance a source with a flux density brighter than or equal to 6 mJy within $r=50^{\prime \prime}$ from J164502 is given by $P=1-e^{-\pi r^{2} N}$, where $N$ is the average surface density of sources with $S_{850 \mu \mathrm{m}} \geq 6 \mathrm{mJy}$ (Downes et al. 1986). Adopting a surface density of $N \sim 400 \mathrm{deg}^{-2}$ (Borys et al. 2003) one finds that $P=0.22$. On that basis we conclude that J164502-SMM1 is most likely unrelated to J164502. 


\section{Analysis \& Discussion}

Table 2 summarises the CO and sub-mm observations of J164502 and J164502-SMM1 as well as the physical quantities derived from them.

\subsection{Molecular gas and dust in J164502}

For an observed velocity-integrated line flux density of the $J+1 \rightarrow J$ CO line, the intrinsic CO line luminosity is given by

$$
\mathrm{L}_{\mathrm{CO}_{\mathrm{J}+1, \mathrm{~J}}}=\frac{\mathrm{c}^{2}}{2 \mathrm{k} \nu_{\mathrm{J}+1, \mathrm{~J}}^{2}} \frac{\mathrm{D}_{\mathrm{L}}^{2}}{1+\mathrm{z}} \int_{\Delta \mathrm{v}} \mathrm{S}_{\nu_{\mathrm{obs}}} \mathrm{dv},
$$

where $D_{L}$ is the luminosity distance, and $\nu_{J+1, J}$ is the rest-frame frequency of the CO transition. Inserting astrophysical units, the equation reads

$$
\mathrm{L}_{\mathrm{CO}_{\mathrm{J}+1, \mathrm{~J}}}=2.43 \times 10^{3}(\mathrm{~J}+1)^{-2}(1+\mathrm{z})^{-1}\left(\frac{\mathrm{D}_{\mathrm{L}}}{\mathrm{Mpc}}\right)^{2}\left(\frac{\int_{\Delta \mathrm{v}} \mathrm{S}_{\nu_{\mathrm{obs}}} \mathrm{dv}}{\mathrm{Jy} \mathrm{km} \mathrm{s}^{-1}}\right),
$$

where $\mathrm{L}^{\prime} \mathrm{CO}_{\mathrm{J}+1, \mathrm{~J}}$ is the so-called pseudo-luminosity which is measured in units of $\mathrm{K} \mathrm{km} \mathrm{s}^{-1} \mathrm{pc}^{2}$. The observed CO $J=1-0$ line flux density for J164502 implies an intrinsic CO luminosity of $\mathrm{L}_{\mathrm{CO}}^{\prime}(1-0)=(7 \pm 1) \times 10^{10} \mathrm{~K} \mathrm{~km} \mathrm{~s}^{-1} \mathrm{pc}^{2}$. From the $\mathrm{CO} J=1-0$ line the molecular gas mass can be found using the scaling relation $M\left(\mathrm{H}_{2}\right)=\mathrm{X}_{\mathrm{CO}} \mathrm{L}_{\mathrm{CO}}^{\prime}(1-0)$ where $X_{\mathrm{CO}} \simeq$ $5 M_{\odot}\left(\mathrm{K} \mathrm{km} \mathrm{s}^{-1} \mathrm{pc}^{2}\right)^{-1}$ is the standard Galactic $\mathrm{CO}-\mathrm{H}_{2}$ conversion factor. The standard conversion factor, $X_{\mathrm{CO}}$ has been calibrated using Giant Molecular Clouds (GMCs) in our Galaxy (e.g. Strong et al. 1988). However, the conditions of the interstellar medium in active starbursting galaxies such as ULIRGs is markedly different from that in our Galaxy, and applying the Galactic conversion factor would result in seriously underestimated gas masses. In particular, the concept of isolated and virialised GMCs breaks down, and instead the bulk of the $\mathrm{CO}$ emission is found to originate from a warm diffuse phase. In effect, this yields a lower conversion factor of $X_{\mathrm{CO}}=0.8 M_{\odot}\left(\mathrm{K} \mathrm{km} \mathrm{s}^{-1} \mathrm{pc}^{2}\right)^{-1}$, more appropriate for such extreme environments (Downes \& Solomon 1998). It is reasonable to assume that in high-redshift starbursts similar conditions prevail and the aforementioned value of $X_{\mathrm{CO}}$ is also adopted for J164502. In doing so we estimate the molecular gas mass present in J164502 to be $M\left(\mathrm{H}_{2}\right)=(6 \pm 1) \times 10^{10} M_{\odot}$. This is in agreement with the $J=2-1$ detection by

Andreani et al. (2000), which yields a molecular gas mass of $\sim 4 \times 10^{10} M_{\odot}$ when converted to the cosmology adopted in this paper.

The CO $J=1-0$ line luminosity and the implied molecular gas mass of J164502 is somewhat lower than those found in the quasars APM 08279+5255 and PSS 2322+1944, and 
in the high redshift radio galaxy $4 \mathrm{C} 60.07\left(\mathrm{~L}_{\mathrm{CO}}^{\prime}(1-0) \sim 10^{11} \mathrm{~K} \mathrm{~km} \mathrm{~s}^{-1} \mathrm{pc}^{2}\right.$ and $M\left(\mathrm{H}_{2}\right) \sim$ $10^{11} M_{\odot}$ - see Papadopoulos et al. 2001; Carilli et al. 2002; Greve et al. in preparation). This is not surprising since these objects are among the most luminous systems in the Universe, and might therefore be expected to contain more gas. Solomon et al. (1997) observed CO $J=1-0$ in a sample of 37 local ULIRGs out to a redshift of $z=0.3$, and found an average $J=1-0$ luminosity of $\mathrm{L}_{\mathrm{CO}}^{\prime}(1-0) \simeq 8 \times 10^{9} \mathrm{~K} \mathrm{~km} \mathrm{~s}^{-1} \mathrm{pc}^{2}$ which is roughly the luminosity of Arp 220 and Mrk 231. The scatter on this result was only 30 per cent. A more recent survey of $\mathrm{CO} J=1-0$ observations of a complete sample of 60 ULIRGs selected from the SCUBA Local Universe Galaxy Survey (SLUGS - Dunne et al. (2000)) yielded similar results (Yao et al. 2003). Hence, we find that J164502 has a CO $J=1-0$ luminosity and consequently a molecular gas mass which is about an order of magnitude larger than is found for local ULIRGs.

The dynamical mass of the system can be calculated from the observed size and line width of the source and assuming the gas is distributed in a disk with diameter $L$ in Keplerian rotation, in which case it can be shown that the dynamical mass is given by

$$
\begin{aligned}
M_{d y n} & \simeq \frac{\Delta V_{F W H M}^{2} L}{2 \alpha_{d} G} \frac{1}{\sin ^{2} i} \\
& =1.16 \times 10^{9}\left(\frac{\Delta V_{F W H M}}{100 \mathrm{~km} \mathrm{~s}^{-1}}\right)^{2} \times\left(\frac{R}{\mathrm{kpc}}\right) \frac{1}{\sin ^{2} i} M_{\odot},
\end{aligned}
$$

where $i$ is the inclination angle of the disk, and $\alpha_{d}$ is a correction factor of order unity (Bryant \& Scoville 1996). Adopting a line width of $\Delta V_{F W H M}=400 \mathrm{~km} \mathrm{~s}^{-1}$ (Andreani et al. 2000), a source size of $\theta \lesssim \sqrt{4.5^{\prime \prime} \times 3.0^{\prime \prime}}=3.7^{\prime \prime}$, which corresponds to a maximum linear diameter of $L \sim 34 \mathrm{kpc}$ at a redshift of $z=1.44$ we estimate the enclosed dynamical mass within the CO-emitting region to be $M_{d y n} \lesssim 6.3 \times 10^{11}(\sin i)^{-2} M_{\odot}$. The inferred ratio of the molecular-to-dynamic mass for the system as a whole is then $M\left(\mathrm{H}_{2}\right) / \mathrm{M}_{\mathrm{dyn}} \gtrsim 0.1 \sin ^{2} \mathrm{i}$. Hence, geometrical factors aside, the amount of molecular gas accounts for at least 10 per cent of the total dynamical mass within the CO-emitting region. The dynamical mass should be considered an upper limit on the total amount of molecular gas present in J164502, and the two mass estimates would only coincide if the $\mathrm{CO}$ emission was concentrated within the inner $\sim 3 \mathrm{kpc}$. Due to the higher spatial resolution and the fact that the $J=1-0$ line provides an unbiased estimate of $M\left(\mathrm{H}_{2}\right)$, our constraint on the molecular-to-dynamical mass ratio should be an improvement over that of Andreani et al. (2000). A gas mass fraction of $\gtrsim 10$ per cent is compatible with the range of gas mass fractions found for local ULIRGs (Downes \& Solomon 1998) yet, unlike the latter, it is distributed over significantly larger scales.

The extrapolated synchroton radiation flux at $850-\mu \mathrm{m}$ is less than $3 \mu \mathrm{Jy}$, and it is there- 
fore safe to assume that the observed $850-\mu \mathrm{m}$ flux from J164502 is dominated by thermal dust emission, with the radio synchroton emission contributing a negligible amount. The dust mass can therefore be estimated using

$$
M_{d}=\frac{S_{\nu_{\mathrm{obs}}} D_{L}^{2}}{(1+z) \kappa_{d}\left(\nu_{\mathrm{rest}}\right)}\left[B\left(\nu_{\mathrm{rest}}, T_{d}\right)-B\left(\nu_{\mathrm{rest}}, T_{c m b}(z)\right]^{-1},\right.
$$

where $\nu_{\text {rest }}=\nu_{\text {obs }}(1+z)$ is the rest-frame frequency, $T_{c m b}(z)$ is the CMB temperature at redshift $z$, and $\kappa_{d} \propto \nu^{\beta}$ is the dust absorption coefficient. The emissivity index, $\beta$, depends on the dust temperature, $T_{d}$, (Dunne et al. 2000) both of which are poorly constrained for high-redshift objects - typical values are $\beta=1-2$. Dey et al. (1999) found the spectral energy distribution (SED) of J164502 to be well described by an optically thin modified black body law with a dust temperature of $T_{d}=40 \mathrm{~K}$ and an emissivity law of $\beta=1.5$, and we adopt those values here. In doing so we estimate the dust mass to be $M_{\text {dust }} \simeq 9 \times 10^{8} M_{\odot}$, where we have used a dust absorption coefficient of $\kappa_{d}\left(\nu_{\text {rest }}\right)=0.11\left(\nu_{\text {rest }} / 353 \mathrm{GHz}\right)^{\beta} \mathrm{m}^{2} \mathrm{~kg}^{-1}$ after Hildebrand (1983). The uncertainty in $k_{d}$ is large, so we present this estimate for comparison only. We can estimate the total far-IR luminosity by integrating the thermal spectrum

$$
L_{F I R}=4 \pi M_{d} \int_{0}^{\infty} \kappa_{d}(\nu) B\left(\nu, T_{d}\right) d \nu
$$

which yields a total far-IR luminosity of $L_{F I R} \simeq 9 \times 10^{12} L_{\odot}$. This almost puts J164502 in the class of hyperluminous infrared galaxies (HyLIRGs) which have $L_{F I R} \gtrsim 10^{13} L_{\odot}$. The dust masses and far-IR luminosities typically found in local ULIRGs are $M_{\text {dust }} \simeq 10^{7-8} M_{\odot}$ and $L_{F I R} \gtrsim 10^{12} L_{\odot}$ (Sanders \& Mirabel 1996; Dunne et al. 2000), i.e. nearly an order of magnitude smaller than what we find for J164502. It is comparable, however, to what is found in high-redshift QSOs and HzRGs (Omont et al. 2003; Archibald et al. 2001) and, perhaps more importantly, similar to the dust masses and far-IR luminosities found in submm-selected dust-enshrouded starbursts at high redshifts (Ivison et al. 1998, 2000). From the above we estimate the gas-to-dust mass ratio of $\mathrm{J} 164502$ to be $M\left(\mathrm{H}_{2}\right) / \mathrm{M}_{\text {dust }} \simeq 67$ which is well within the range of 50-100 found for local ULIRGs. Note, however, that significant uncertainty is attached to the normalisation of $\kappa_{d}$, and values differing by as much as a factor of two has been reported (Draine \& Lee 1984; Mathis \& Whiffen 1989), which in turn could lead to a similar change in the dust mass but not in $L_{F I R}$ which is independent of the normalisation value of the dust absorption coefficient. Using $\beta=2.0$ instead of 1.5 would decrease the dust mass by nearly a factor of two, but increase $L_{F I R}$ by a similar amount.

If the bulk of the far-IR emission can be ascribed to starburst activity the corresponding star formation rate is given by

$$
S F R \simeq L_{F I R} 10^{-10} \delta_{I M F} \delta_{S B} L_{\odot} M_{\odot} \mathrm{yr}^{-1},
$$


where $\delta_{I M F} \sim 1-6$ is a function of the initial mass function, and $\delta_{S B}$ is the fraction of the FIR emission which is heated by the starburst (Omont et al. 2001). Assuming a conservative value of $\delta_{I M F}=1$, we estimate the starformation rate in $\mathrm{J} 164502$ to be $S F R \simeq 900 \delta_{S B} M_{\odot} \mathrm{yr}^{-1}$. It is possible that J164502 harbours an AGN in its center, so a significant fraction of the farIR luminosity could be due to dust being heated by the AGN and not by the starburst. The derived value for the SFR could therefore be overestimated. However, the narrow linewidths seen in optical and NIR spectra of J164502 (Dey et al. 1999) favours young hot stars over an AGN as the main source of energy. Furthermore, as pointed out by Dey et al. (1999), J164502 deviates from the $60 \mu \mathrm{m}-6 \mathrm{~cm}$ relation obeyed by local star-forming galaxies by having almost an order of magnitude less radio emission at $6 \mathrm{~cm}$, which is unlikely if an AGN dominated the energetics of the system (Dey \& van Breugel 1994). In addition, it is found that the bulk of the far-IR emission from local ULIRGs is powered by starburst activity even though an AGN is present (Downes \& Solomon 1998). Finally, if the sub-mm emission is extended it would strongly suggest that the main power source for the far-IR emission is a massive starburst since it is difficult to imagine the AGN heating the dust on scales of tens of kpc. The large quantity of molecular gas revealed by the $\mathrm{CO}$ detections in this system could provide the necessary fuel for such a massive starburst for a period of $\sim 10^{7}$ years.

The efficiency of star formation should be measured relative to the amount of molecular gas available to form stars. Such a measure of the star-formation efficiency is the rate of star formation per solar mass of molecular hydrogen, i.e. $L_{F I R} / M\left(\mathrm{H}_{2}\right)$. For J164502 we find $L_{F I R} / M\left(\mathrm{H}_{2}\right) \simeq 150 L_{\odot} M_{\odot}{ }^{-1}$. Probably a better gauge of the star-formation efficiency is the continuum-to-line ratio $L_{F I R} / L_{\mathrm{CO}}^{\prime}(1-0)$ since it is independent of $X_{\mathrm{CO}}$. Giant Molecular Cloud (GMCs) in our Galaxy typically have values of $\sim 15$ and similar ratios are found in nearby spirals (Mooney \& Solomon 1988). Starburst galaxies and ULIRGs have $L_{F I R} / L_{\mathrm{CO}}^{\prime}(1-0)$ ratios which are 10 times higher than this, ranging in values from 80 to 250 with a median of $160 L_{\odot}\left(\mathrm{K} \mathrm{km} \mathrm{s}^{-1} \mathrm{pc}^{2}\right)^{-1}$ (Solomon et al. 1997). For J164502 we find $L_{F I R} / L_{\mathrm{CO}}^{\prime}(1-0) \simeq 129 L_{\odot}\left(\mathrm{K} \mathrm{km} \mathrm{s}^{-1} \mathrm{pc}^{2}\right)^{-1}$, i.e. in line with what is found for local ULIRGs. Recent studies of large samples of ULIRGs at low and intermediate redshifts have shown that the $L_{F I R} / L_{\mathrm{CO}}^{\prime}(1-0)$ ratio increases with increasing $L_{F I R}$ (Young et al. 1986; Tutui et al. 2000; Yao et al. 2003). Such behaviour can be explained if the more far-IR luminous galaxies, in addition to having more dust and gas which would just continue the linear relation between $L_{F I R}$ and $L_{\mathrm{CO}}^{\prime}(1-0)$, have higher dust temperatures due to extra heating by an AGN or a higher star-formation efficiency. Assuming that there is no contribution to $L_{F I R}$ from an AGN, the derived $L_{F I R} / L_{\mathrm{CO}}^{\prime}(1-0)$-ratio should probably be taken as a lower limit on the star-formation efficiency since in the extreme starburst regions dominating the emission from ULIRGs, the bulk of the CO luminosity comes from a diffuse inter-cloud medium, rather than from the dense gas gravitationally bound in clouds where the stars are 
formed (Downes et al. 1993; Solomon et al. 1997). The dense gas is better traced by HCN, and as shown by Gao et al. (1999) the $L_{F I R} / L_{\mathrm{HCN}}$-ratio is the same for GMCs to ULIRGs, indicating that anywhere in the Universe only the dense gas is relevant to star-formation. In ULIRGs the dense/diffuse gas mass ratio (roughly quantified by $L_{\mathrm{HCN}} / L_{\mathrm{CO}}$ ) is particularly high which is not a surprise given their merger status and the way the gas responds to a merger.

\subsection{Excitation conditions of the molecular gas in $\mathrm{J} 164502$}

The velocity/area-averaged brightness temperature ratio between $\mathrm{CO} J+1 \rightarrow J$ and $\mathrm{CO} 1 \rightarrow 0$ is

$$
r_{J+1, J}=\frac{T_{b}(J+1 \rightarrow J)}{T_{b}(1 \rightarrow 0)}=\frac{L^{\prime}(J+1 \rightarrow J)}{L^{\prime}(1 \rightarrow 0)} .
$$

Andreani et al. (2000) estimated the CO $J=2-1$ and $J=5-4$ luminosities to be $4 \times$ $10^{10}$ and $7 \times 10^{9} \mathrm{~K} \mathrm{~km} \mathrm{~s}^{-1} \mathrm{pc}^{2}$, respectively, where we have converted the luminosities to the cosmology adopted here. The line ratios estimated from eq. 8 are $r_{21}=0.6 \pm 0.2$ and $r_{54}=0.10 \pm 0.05$. The majority of high-redshift systems detected in $\mathrm{CO}$ to date are strongly gravitationally lensed. This makes estimating the excitation conditions in such systems complicated since differential magnification of the high-J lines compared to lower transitions may bias the line ratios significantly. There is nothing to suggest that J164502 is gravitationally lensed, and the above derived line ratios ought to represent the intrinsic excitation conditions of the gas, albeit still averaged over the entire galaxy.

We used the observed line ratios of $r_{21}=0.6 \pm 0.2$ and $r_{54}=0.10 \pm 0.05$ as constraints to a Large Velocity Gradient (LVG) code in order to gain some insight on the bulk properties of the molecular gas in J164502. A lower limit on gas temperature $\mathrm{T}_{\text {kin }}=40 \mathrm{~K}$ is assumed since this value is deduced for the bulk of the dust (Dey et al. 1999) and $T_{\text {kin }} \gtrsim T_{\text {dust }}$ is expected for FUV-heated gas/dust (e.g. Tielens \& Hollenbach 1999). A wide range of temperatures $\left(\mathrm{T}_{\text {kin }}=60-90 \mathrm{~K}\right)$ offers an acceptable fit to the aforementioned line ratios, with $\mathrm{T}_{\text {kin }}=70 \mathrm{~K}$ being the optimal value. This wide range is due to the poor constraints offered by only two line ratios while the high gas temperatures may reflect the UV-intense environment of the molecular gas in J164502 and/or turbulent motions heating only the gas, a situation that has already been noted for molecular gas in the Galactic center (Rodriguez-Fernandez et al. 2001).

A common feature of all the LVG solutions is the low gas densities $\mathrm{n}\left(\mathrm{H}_{2}\right) \sim 300 \mathrm{~cm}^{-3}$. This has already been noted when only the CO $(5-4) /(2-1)$ line ratio was available (Papadopoulos \& Ivison 2002) and is a property often found for the bulk of the gas in 
extreme starbursts (e.g. Aalto et al. 1995; Downes \& Solomon 1998). Interestingly for such low gas densities all CO $J+1 \rightarrow J$ transitions with $J+1>5$ have flux density ratios with respect to the lowest $1-0$ transition of $S(J+1-J) / S(1-0)=(J+1)^{2} r_{\mathrm{J}+1} \mathrm{~J} \lesssim 0.25$ for any plausible gas temperature $\left(\mathrm{T}_{\text {kin }} \sim 40-100 \mathrm{~K}\right)$, thus no longer offering the advantage of a higher flux density with respect to the latter.

As a consequence, any estimate of the $\mathrm{H}_{2}$ mass based on the assumption of optically thick, thermalised high-J CO lines could be severely underestimated. Using the $5-4$ line in J164502 only, and assuming it is optically thick and thermalised $\left(r_{54} \sim 1\right)$, one would find a molecular gas mass which is an order of magnitude lower than that inferred from the $J=1-0$ line. There is no significant flux advantage between the $2-1$ and $1-0$ lines, since $S_{\mathrm{CO}}(2-1) / S_{\mathrm{CO}}(1-0)=4 T_{b}(2-1) / T_{b}(1-0)>1$ even for a very subthermal ratio of $r_{21}=0.3$, say. This is consistent with the fact that we find the molecular masses derived from the two lines to be similar (section 4.1). The above demonstrates the importance of using low-J CO lines in order to infer the amount of molecular gas in a galaxy. This is particularly true at redshifts beyond 3 , since at those redshifts the current mm-interferometers can only hope to detect $\mathrm{CO} J+1 \rightarrow J, J>2$, and the the excitation bias of the high-J lines could become very severe.

\subsection{Comparison with high-redshift sub-mm galaxies}

Although J164502 was originally selected as an ERO and not a (sub)millimetre galaxy, subsequent observations at optical/IR and sub-mm wavelengths have shown that it can in fact be considered a typical sub-mm galaxy (Dey et al. 1999; see also Smail et al. 1999). Our detection of CO $J=1-0$ in J164502 is therefore the first detection of this transition in a galaxy which is thought to be similar to the SCUBA population of dust-enshrouded galaxies at high redshifts. J164502 has a far-IR and CO luminosity about ten times that of the average values found in ULIRGs, and the amount of molecular gas present in J164502 is comparable to the median gas mass $\left(\sim 2 \times 10^{10} M_{\odot}\right)$ of the five SCUBA galaxies detected in CO to date (Neri et al. 2003), although a thorough characterisation of the gas content of this population has to await CO observations of a large, unbiased sample of submm-selected galaxies. Hence, the observations presented here further supports that J164502 is more similar in its properties to sub-mm galaxies than a high-redshift analogue of Arp 220.

Recently, a subject of some debate has been whether the $\mathrm{CO}$ emission detected from high-redshift sub-mm galaxies originates from a huge, massive resevoir of molecular gas $\sim 10 \mathrm{kpc}$ in size or from a much more compact circumnuclear disk, typically of radius $r \sim$ 100 pc, as seen in local ULIRGs such as Arp 220 (Ivison et al. 2001; Genzel et al. 2003; 
Downes \& Solomon 2003). If, as the observations seem to suggest, the starburst in J164502 is extended over several tens of $\mathrm{kpc}$ that would set apart from the local ULIRG population and point toward the former scenario.

TRG acknowledges support from the Danish Research Council and from the EU RTN Network POE. PPP acknowledges a Marie Curie Individual Fellowship HPMT-CT-2000-

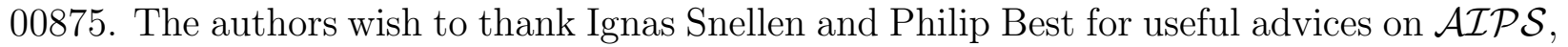
and Omar Almaini for kindly providing us with the reduced UKIRT UFTI K-band image of J164502+4626.4. We also thank Jason Stevens and Andy Taylor for useful discussions.

\section{REFERENCES}

Aalto, S., Booth, R. S., Black, J. H. \& Johansson, L. E. B. 1995, A\&A, 300, 369.

Andreani, P., Cimatti, A., Loinard, L. \& Röttgering, H. 2000, A\&A, 354, L1.

Archibald, E. N., Dunlop, J. S., Hughes, D. H., Rawlings, S., Eales, S. A. \& Ivison, R. J. 2001, MNRAS, 323, 417.

Bertoldi, F., Menten, K.M., Kreysa, E., Carilli, C.L. \& Owen, F. 2001, 24th meeting of the IAU, Manchester, England (astro-ph 0010553).

Borys, C., Chapman, S., Halpern, M. \& Scott, D. 2003, MNRAS , in press, astro-ph/0305444.

Brown, R.L. \& vanden Bout, P.A. 1991, A\&A, 102, 1956.

Bryant, P.M. \& Scoville, N.Z. 1996, ApJ, 457, 678.

Carilli, C.L., Cox, P., Bertoldi, F., Menten, K.M., Omont, A., Djorgovski, S.G., Petric, A., Beelen, A., Isaak, K.G. \& McMahon, R.G. 2002, ApJ, 575, 145.

Chapman, S.C., Blain, A.W., Ivison, R.J. \& Smail, I. 2003, Nature, accepted.

Cimatti, A., Andreani, P., Röttgering, H. \& Tilanus, R. 1998, Nature, 392, 895.

Dey, A. \& van Breugel, W. 1994, "Mass Transfer Induced Activity in Galaxies", Cambridge Univ. Press, ed. Shlosman, I.

Dey, A., Graham, J. R., Ivison, R. J., Smail, I., Wright, G. S. \& Liu, M. C. 1999, ApJ, 519, 610.

Downes, A. J. B., Peacock, J. A., Savage, A., Carrie, D. R. 1986, MNRAS, 218, 31. 
Downes, D., Solomon, P.M. \& Radford, S.J.E. 1993, ApJ, 414, L13.

Downes, D. \& Solomon, P.M. 1998, ApJ, 507, 615.

Downes, D. \& Solomon, P.M. 2003, ApJ, 582, 37.

Draine, B. \& Lee, H. 1984, ApJ, 285, 89.

Dunlop, J., Peacock, J., Spinrad, H., Dey, A., Jimenez, R., Stern, D. \& Windhorst, R., Nature, 381, 581 .

Dunne, L., Eales, S, Edmunds, M., Ivison, R.J., Alexander \& P. Clements, D.L. 2000, MNRAS, 315, 115.

Frayer, D.T. 1996, PhD Thesis, University of Virginia.

Frayer, D.T., Ivison, R.J., Scoville, N.Z., Yun, M., Evans, A.S., Smail, I., Blain, A.W. \& Kneib, J.-P. 1998, ApJ, 506, 7.

Frayer, D.T., Ivison, R.J., Scoville, N.Z., Evans, A.S., Yun, M.S., Smail, I., Barger, A.J., Blain, A.W. \& Kneib, J.-P. 1999, ApJ, 514 L13.

Gao, Y., Solomon, P.M. \& Philip, M. 1999, ApJ, 512, 99.

Genzel, R., Baker, A.J., Tacconi, L.J., Lutz, D., Cox, P., Guilloteau, S. \& Omont, A. 2003, ApJ, 584, 633.

Graham, J. R. \& Dey, A. 1996, ApJ, 471, 720.

Hildebrand, R. 1983, QJRAS, 24, 267.

Holland, W. S., Robson, E. I., Gear, W. K., Cunningham, C. R., Lightfoot, J. F., Jenness, T., Ivison, R. J., Stevens, J. A., Ade, P. A. R., Griffin, M. J., Duncan, W. D., Murphy, J. A. \& Naylor, D. A. 1999, MNRAS, 303, 659.

Hu, E. M. \& Ridgway, S. E. 1994, AJ, 107, 1303.

Hughes, D.H., Serjeant, S., Dunlop, J., Rowan-Robinson, M., Blain, A., Mann, R. G., Ivison, R., Peacock, J., Efstathiou, A., Gear, W., Oliver, S., Lawrence, A., Longair, M., Goldschmidt, P. \& Jenness, T. 1998, Nature, 394, 241.

Ivison, R.J., Papadopoulos, P.P., Seaquist, E.R. \& Eales, S.A. 1996, MNRAS, 278, 669.

Ivison, R.J., Smail, I., Le Borgne, J.-F., Blain, A.W., Kneib, J.-P., Bezecourt, J., Kerr \& T.H., Davies, J.K. 1998, MNRAS, 298, 583. 
Ivison, R.J., Smail, I., Barger, A.J., Kneib, J.-P., Blain, A.W., Owen, F.N., Kerr, T.H. \& Cowie, L.L. 2000, MNRAS, 315, 209.

Ivison, R.J., Smail, I., Frayer, D.T., Kneib, J.-P. \& Blain, A.W. 2001, ApJ, 561, L45.

Ivison, R.J., Greve, T.R., Smail, I., Dunlop, J., Roche, N., Scott, S., Page, M., Stevens, J., Almaini, O., Blain, A., Willott, C., Fox, M., Gilbank, D., Serjeant, S. \& Hughes, D. 2002, MNRAS, 337, 1.

Kreysa, E., Gemuend, H.-P., Gromke, J., Haslam, C.G., Reichertz, L., Haller, E.E., Beeman, J.W., Hansen, V., Sievers, A. \& Zylka, R. 1998, Proc SPIE 3357, 319.

Mathis, J. \& Whiffen, G. 1989, ApJ, 341, 808.

Monet, D., Bird A., Canzian, B., Dahn, C., Guetter, H., Harris, H., Henden, A., Levine, S., Luginbuhl, C., Monet, A. K. B., Rhodes, A., Riepe, B., Sell, S., Stone, R., Vrba, F. \& Walker, R. 1998, USNO-SA2.0 (U.S. Naval Observatory, Washington DC).

Mooney, T. \& Solomon, P.M. 1988, ApJ, 334, L51.

Moriondo, G., Cimatti, A., Daddi, E. 2000, A\&A, 364, 26.

Neri, R., Genzel, R., Ivison, R.J., Bertoldi, F., Blain, A.W., Chapman, S.C., Cox, P., Greve, T.R., Omont, A. \& Frayer, D.T. 2003, ApJ, submitted.

Omont, A., Cox, P., Bertoldi, F., McMahon, R., Carilli, C., \& Isaak, K. G. 2001, A\&A, 374, 371.

Omont, A., Beelen, A., Bertoldi, F., Cox, P., Carilli, C. L., Priddey, R. S., McMahon, R. G. \& Isaak, K. G. 2003, A\&A, 398, 857.

Papadopoulos, P. P., Röttgering, H. J. A., van der Werf, P. P., Guilloteau, S., Omont, A., van Breugel, W. J. M. \& Tilanus, R. P. J. 2000, ApJ, 528, 626.

Papadopoulos, P.P., Ivison, R.J., Carilli, C.L. \& Lewis, G. 2001, Nature, 409, 58.

Papadopoulos, P. P. \& Ivison, R.J. 2002, ApJ, 564, L9.

Rodriguez-Fernandez, N. J., Martin-Pintado, J., Fuente, A., de Vicente, P., Wilson, T. L., \& Hüttemeister, S. 2001, A\&A, 365, 174.

Sanders, D.B., Scoville, N.Z. \& Soifer, B.T. 1991, ApJ, 370, 158.

Sanders, D.B. \& Mirabel, I.F. 1996, ARA\&A, 34, 749. 
Smail, I., Ivison, R.J. \& Blain, A.W. 1997, ApJ, 490, L5.

Smail, I., Ivison, R.J., Kneib, J.-P., Cowie, L.L., Blain, A.W., Barger, A.J., Owen, F.N. \& Morrison, G. 1999, MNRAS, 308, 1061.

Solomon, P.M., Downes, D., Radford, S.J.E. \& Barrett, J.W. 1997, ApJ, 478, 144.

Strong, A. W., Bloemen, J. B. G. M., Dame, T. M., Grenier, I. A., Hermsen, W., Lebrun, F., Nyman, L.-A., Pollock, A. M. T. \& Thaddeus, P. 1988, A\&A, 201, 1.

Tielens, D.J. \& Hollenbach, A.G.G.M. 1999, Rev. Modern Physics, 71, 174.

Tutui, Y., Sofue, Y., Honma, M., Ichikawa \& T., Wakamatsu, K.-i. 2000, PASP. 52, 803.

Yao, L., Seaquist, E.R., Kuno, N. \& Dunne, L. 2003, AJ, accepted.

Young, J.S., Schloerb, F.P., Kenney, J.D. \& Lord, S.D. 1986, ApJ, 304, 443. 

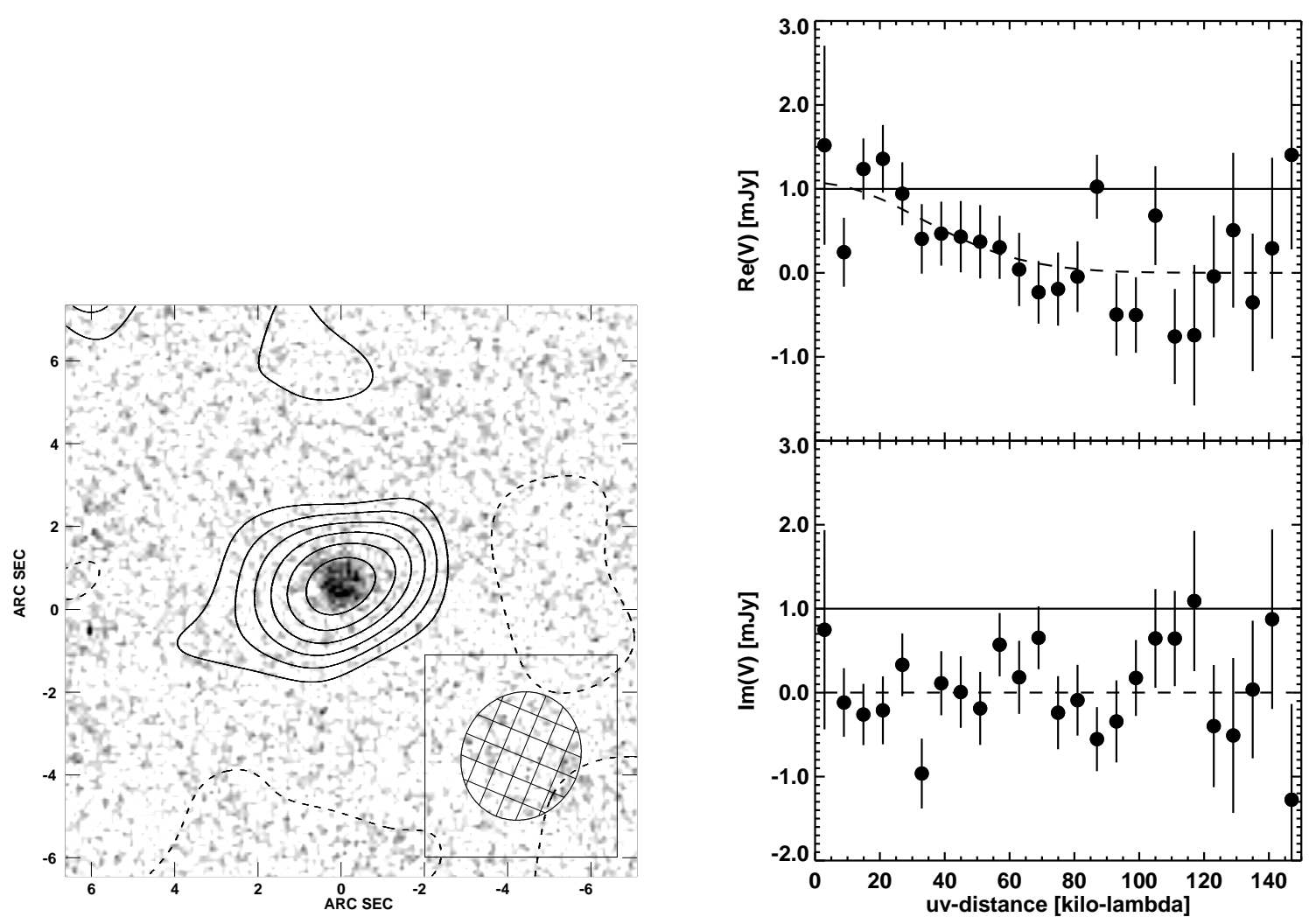

Fig. 1.- Left: Naturally weighted CO $J=1-0$ contour map of J164502 overlaid on the UKIRT UFTI K-band image. The resolution of the CO map is $3.1^{\prime \prime} \times 2.8^{\prime \prime}$ at $P A=157^{\circ}$, see the insert. The contours are $-2,2,3,4,5,6$, and $7 \sigma$ where $\sigma=0.1 \mathrm{mJybeam}^{-1}$. Right: The real (top panel) and imaginary (bottom panel) part of the complex visibilities vs. baseline. The visibilities have been binned. The solid line represents a point source with flux density of $1.0 \mathrm{mJy}$. The dashed curves represent a least-square fit of a Gaussian source model to the visibilities. 

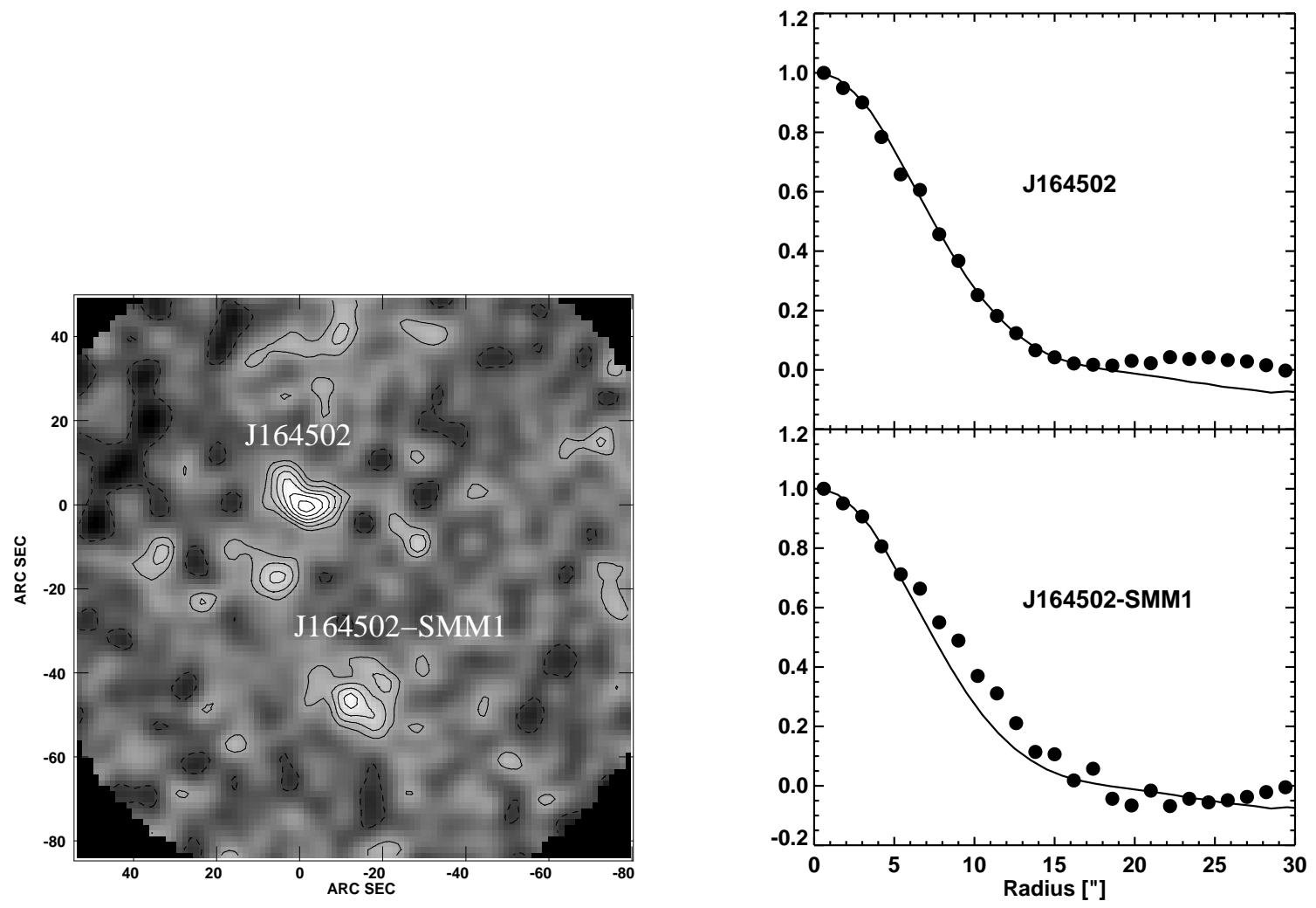

Fig. 2.- Left: SCUBA $850-\mu \mathrm{m}$ jiggle-maps of J164502. The resolution is $F W H M=14^{\prime \prime}$, and the rms noise level is $1.6 \mathrm{mJy}_{\text {beam }}{ }^{-1}$. Contours start at $2 \sigma$. J164502-SMM1 denotes a statistically significant detection of $850-\mu \mathrm{m}$ emission about $50^{\prime \prime}$ south of J164502. Weak emission is also seen 20" south-east of J164502. Right: Radial profiles of J164502 and J164502-SMM1 (filled circles) compared with the radial profile of the PSF (solid curve). 
Table 1. VLA Observations

\begin{tabular}{lccc}
\hline \hline Date & Configuration & Frequency, (GHz) & Integration time, (hrs) \\
\hline 2001 Oct 9 & D & $47.235,47.285$ & 5.2 \\
2002 Oct 26 & C & $47.235,47.285$ & 2.9 \\
2002 Nov 14 & C & $47.235,47.285$ & 2.4 \\
2002 Dec 24 & C & $47.235,47.285$ & 2.6 \\
2003 Feb 21 & D & $47.235,47.285$ & 2.6 \\
2003 Mar 1 & D & $47.235,47.285$ & 3.8 \\
\hline
\end{tabular}


Table 2. Observed properties of J164502

\begin{tabular}{lcc}
\hline \hline \multicolumn{1}{c}{ Parameter } & $\mathrm{J} 164502$ & $\mathrm{~J} 164502-\mathrm{SMM} 1$ \\
\hline$\alpha(\mathrm{J} 2000)$ & $16: 45: 02.26$ & $16: 45: 01.20$ \\
$\delta(\mathrm{J} 2000)$ & $+46: 26: 26.50$ & $+46: 25: 39.0$ \\
$z_{\mathrm{CO}}$ & 1.439 & \\
$\int_{\Delta V} S_{\mathrm{CO}(1-0)} d V\left[\mathrm{Jy} \mathrm{km} \mathrm{s}^{-1}\right]$ & $0.6 \pm 0.1$ & $<0.2$ \\
$S_{850 \mu \mathrm{m}}[\mathrm{mJy}]$ & $8 \pm 2^{\mathrm{a}}$ & $6 \pm 2^{\mathrm{b}}$ \\
$\mathrm{L}_{\mathrm{CO}}^{\prime}(1-0)\left[\mathrm{K} \mathrm{km} \mathrm{s}^{-1} \mathrm{pc}^{-1}\right]$ & $(7 \pm 1) \times 10^{10}$ & $<2 \times 10^{10 \mathrm{c}}$ \\
$L_{F I R}\left[L_{\odot}\right]$ & $9 \times 10^{12}$ & $7 \times 10^{12 \mathrm{c}}$ \\
\hline
\end{tabular}

${ }^{a}$ The average of $7.5 / 7.9 / 7.3 / 9.8 \mathrm{mJy}$ which was the flux densities obtained using the four different methods described in section 3 .

${ }^{\mathrm{b}}$ The average of $6.7 / 6.5 / 6.0 / 6.5 \mathrm{mJy}$ which was the flux densities obtained using the four different methods described in section 3 .

${ }^{\mathrm{c}}$ This value assumes that J164502-SMM1 has a redshift of $z=$ 1.439 . 